\title{
Effects of pulmonary rehabilitation program on amyotrophic lateral sclerosis: A meta-analysis of randomized controlled trials
}

\author{
Chien-Ling Su $\mathrm{Su}^{\mathrm{a}, \mathrm{b}, \mathrm{h}}$, Ka-Wai Tam ${ }^{\mathrm{c}, \mathrm{d}, \mathrm{e}}$, Tien-Pei Fang ${ }^{\mathrm{f}, \mathrm{g}}$, Ling-Ling Chiang ${ }^{\mathrm{a}, \mathrm{h}}$ and Hui-Chin Chen ${ }^{\mathrm{f}, *}$ \\ ${ }^{a}$ Department of Thoracic Internal Medicine, Taipei Medical University-Shuang Ho Hospital, New Taipei City, \\ Taiwan \\ ${ }^{\mathrm{b}}$ Department of Physical Therapy, Shu-Zen Junior College of Medicine and Management, Kaohsiung City, Taiwan \\ ${ }^{\mathrm{c}}$ Division of General Surgery, Department of Surgery, Shuang Ho Hospital, Taipei Medical University, \\ New Taipei City, Taiwan \\ ${ }^{\mathrm{d}}$ Division of General Surgery, Department of Surgery, School of Medicine, College of Medicine, Taipei Medical \\ University, Taipei, Taiwan \\ ${ }^{\mathrm{e}}$ Cochrane Taiwan, Taipei Medical University, Taipei, Taiwan \\ ${ }^{\mathrm{f}}$ Department of Respiratory Care, Chang Gung University of Science and Technology, Chiayi, Taiwan \\ ${ }^{\mathrm{g}}$ Department of Respiratory Therapy, Chiayi Chang Gung Memorial Hospital, Chiayi, Taiwan \\ ${ }^{\mathrm{h}}$ School of Respiratory Therapy, Taipei Medical University, Taipei, Taiwan
}

Received 9 February 2021

Accepted 23 February 2021

\begin{abstract}
.
BACKGROUND: Patients with amyotrophic lateral sclerosis (ALS) develop respiratory failure and progressive muscle weakness. The effects of pulmonary rehabilitation on the lung function of patients with ALS are unclear.

OBJECTIVE: Through this meta-analysis of randomized controlled trials (RCTs), we evaluated the effects of pulmonary rehabilitation, such as type of treatment, on patients with ALS and compared the effectiveness of this treatment.

METHODS: PubMed, EMBASE, Web of Science, and Cochrane databases were searched until December 2020. The methodological quality of each study was assessed using the updated Cochrane Risk of Bias tool (RoB 2.0). Data were analyzed using Review Manager version 5.4 (Cochrane Collaboration, Oxford, England), and the meta-analysis was performed in accordance with Preferred Reporting Items for Systematic reviews and Meta-Analysis (PRISMA) guidelines.

RESULTS: Of 2168 articles, 10 trials were reviewed; among these trials, two focused on respiratory training and eight on physical exercise, three of which involved a combination of aerobic and resistance training. Our meta-analysis demonstrated no difference in the ALSFRS-R score and \%FVC among patients with ALS.
\end{abstract}

\footnotetext{
*Address for correspondence: Hui-Chin Chen, Department of Respiratory Care, Chang Gung University of Science and Technology, No.2, Sec. W., Jaipur Rd., Puzo City, Chiayi County 61363,
}

Taiwan. Tel.: +886 53628800 /Ext. 2236; Fax: +886 5 3628866; E-mail: claire623@hotmail.com. 
CONCLUSIONS: Respiratory training or physical exercise did not significantly affect the ALSFRS-R score and \%FVC of patients with ALS. At 12 months after intervention, the ALSFRS-R score in the physical exercise group was higher than that in the usual care group. Further clinical trials are warranted to develop approaches for improving the lung function of patients with ALS.

Keywords: Rehabilitation, amyotrophic lateral sclerosis, respiratory training, physical exercise

\section{Introduction}

Amyotrophic lateral sclerosis (ALS) is a progressive neurodegenerative disease that may affect any voluntary muscle, including both upper and lower motor muscles. The goal of treatment for ALS is to improve symptoms and provide supportive care as well to improve quality of life and prolong patient survival (van Es et al., 2017). ALS management focuses on pharmacological treatments, breathing support (including noninvasive ventilation or invasive ventilation), physical therapy, nutrition, and end-of-life care. Thus, physical therapy and exercise prescription play important roles in the rehabilitation of patients with ALS by delaying the loss of strength, maintaining endurance, limiting pain, and promoting functional independence. A previous study reported that resistance exercises provide significantly better functional benefits, as measured by the Amyotrophic Lateral Sclerosis Functional Rating Scale (ALSFRS); however, no differences were found in forced vital capacity (FVC) at 3 or 6 months after intervention (Dal Bello-Haas, et al., 2007).

Respiratory failure and progressive muscle weakness are common concerns in patients with ALS. Respiratory muscle weakness results in ineffective cough; decreased inspiratory muscle strength may lead to alveolar hypoventilation, ventilationperfusion mismatch, and further respiratory muscle fatigue, which may lead to respiratory failure and even death. On average, death may occur within 3 years of diagnosis (Brown \& Al-Chalabi, 2017). The exact cause of this disease is unknown, and it has a genetic probability of 5\%-10\% (Kiernan, et al., 2011). No cure for ALS has been reported thus far.

Pulmonary rehabilitation can improve lung function, improve exercise capacity, reduce symptom severity, and improve quality of life, especially in patients with chronic obstructive pulmonary disease (Rochester, et al., 2015). The benefits of pulmonary rehabilitation are reduced dyspnea, reversal of anxiety and depression, enhanced ability to perform activities of daily living, increased exercise ability, and higher quality of life. Previous studies reported that respiratory muscle training improved the ventila- tory function and respiratory strength of patients with multiple sclerosis and ALS but did not affect FVC (Cheah, et al., 2009; Ferreira, et al., 2016; Gosselink, et al., 2000; Pinto \& de Carvalho, 2013). However, the effects of pulmonary rehabilitation on the lung function of patients with ALS are still unclear; clearer treatment and intervention protocols are warranted. Therefore, we conducted a meta-analysis of randomized controlled trials (RCTs) to evaluate the effects of pulmonary rehabilitation, such as type of treatment, on ALS and to compare the effectiveness of this treatment.

\section{Methods}

\subsection{Search strategy}

PubMed, EMBASE, Web of Science, and Cochrane databases were searched until December 2020. The following keywords were used: "rehabilitation or pulmonary rehabilitation" AND "ALS or amyotrophic lateral sclerosis." MeSH terms and their synonyms were used, and no language restriction was applied. This study was registered in the PROSPERO database (CRD42020204884) and adhered to the Preferred Reporting Items for Systematic reviews and Meta-Analyses (PRISMA) checklist (Liberati et al., 2009). Relevant studies were selected independently by three authors (HCC, LLC, and CLS). Any conflicts in study selection were discussed with a fourth author (KWT) and were resolved by reaching a consensus.

\subsection{Study selection}

\subsubsection{Eligibility criteria}

RCTs, trials in patients with ALS, and those with pulmonary rehabilitation interventions were included in this meta-analysis. Nonhuman trials and nonintervention research were excluded. After removing duplicate results, the titles and abstracts were searched to preliminarily identify eligible studies. The full texts were then evaluated to identify eligible studies, which were ultimately included in the metaanalysis. Any disagreements were resolved through discussion with the fourth reviewer (KWT). 


\subsubsection{Data extraction}

Baseline and outcome data were independently extracted by reviewers; the extracted data included study characteristics (first author, publication year, design, inclusion criteria, participants characteristics, and interventions) and outcomes. The individually recorded data by the reviewers were then compared.

\subsubsection{Methodology assessment}

The methodological quality of each study was assessed using the updated Cochrane Risk of Bias tool (RoB 2.0) (Sterne, et al., 2019). The quality was determined by assessing bias in five domains: bias due to the randomization process, bias due to deviations from intended interventions, bias due to missing outcome data, bias in the measurement of the outcome, and bias in the selection of the reported result. The overall risk of bias was classified as high, having some concerns, and low. Three reviewers (HCC, LLC, and TPF) independently assessed the risk of bias, and any differences were resolved through discussion with the fourth reviewer (KWT).

\subsubsection{Outcome assessment}

The primary outcome was the change in the Revised ALS Functional Rating Scale (ALSFRS-R) score, and the secondary outcome was the change in FVC. The ALSFRS-R assesses the severity of ALS as well as respiratory function. ALS measurement involves the evaluation of speech, salivation, swallowing, handwriting, cutting food and handling utensils (with or without gastrostomy), dressing and hygiene, turning in bed and adjusting bed clothes, walking, climbing stairs, dyspnea, orthopnea, and respiratory insufficiency (points are assigned for all 12 measures). The total score ranges from 0 to 48 , with a higher score indicating higher functional status (Cedarbaum, et al., 1999). The ALSFRS-R provides more weight to respiratory symptoms (compared with the original ALSFRS) and is quick and easy to score. FVC is the amount of air that can be forcibly exhaled from the lungs after taking the deepest breath possible, as measured using spirometry. FVC is used to evaluate lung function and measures the effect of lung disease on one's ability to inhale and exhale.

\subsection{Data pooling and statistical analysis}

Data were analyzed using Review Manager version 5.4 (Cochrane Collaboration, Oxford, England), and the meta-analysis was performed in accordance with PRISMA guidelines (Liberati, et al., 2009). Continuous variables are expressed using the mean difference
(MD) and standard MD. The precision of effect sizes was reported as $95 \%$ confidence intervals (CIs). The study-level data were pooled using a random-effects model (DerSimonian \& Laird, 1986). Cochran's $Q$ and $I^{2}$ statistics were calculated to evaluate the statistical heterogeneity and inconsistency of treatment effects, respectively, across trials. Statistical significance was set as $P<.10$ for Cochran's $Q$ tests. Statistical heterogeneity across trials was assessed using the $I^{2}$ test, which quantifies the proportion of total outcome variability across trials. Trials with $I^{2}>50 \%$ were considered to have significant heterogeneity.

\section{Results}

\subsection{Included studies}

A total of 2168 articles were retrieved from the aforementioned databases, of which 1952 articles remained after deleting duplicate records; 231 articles were excluded after examination of their titles and abstracts, and the eligibility of 1721 articles was evaluated. After reviewing the remaining 21 full-text articles, 10 RCTs (Braga, et al., 2018; Clawson, et al., 2018; Dal Bello-Haas, et al., 2007; Drory, et al., 2001; Lunetta, et al., 2016; Pinto, et al., 2012; Merico, et al., 2018; Plowman, et al., 2019; van Groenestijn, et al., 2019; Zucchi, et al., 2019) were included in the analysis. The study screening process is shown in Fig. 1.

\subsection{Characteristics of included studies}

The characteristics of the trials analyzed are displayed in Table 1. Most of the included trials were published from 2016 to 2019 (Braga, et al., 2018; Clawson, et al., 2018; Lunetta, et al., 2016; Merico, et al., 2018; Plowman, et al., 2019; van Groenestijn, et al., 2019; Zucchi, et al., 2019). Of the 10 trials, 2 involved respiratory training interventions (Pinto, et al., 2012; Plowman, et al., 2019) and 8 involved physical exercise interventions (Braga, et al., 2018; Clawson, et al., 2018; Dal Bello-Haas, et al., 2007; Drory, et al., 2001; Lunetta, et al., 2016; Merico, et al., 2018; van Groenestijn, et al., 2019; Zucchi, et al., 2019), 3 of which involved a combination of aerobic exercises and resistance training (Merico, et al., 2018; van Groenestijn, et al., 2019; Zucchi, et al., 2019). The study participants were mostly patients with mild to moderate ALS; most patients were men $(65.5 \%)$, and the average patient age was 60.7 (range, 18-80) years. The minimal ALSFRS-R (0-48) score was $36.6 \pm 6.3$ points (Plowman, et al., 


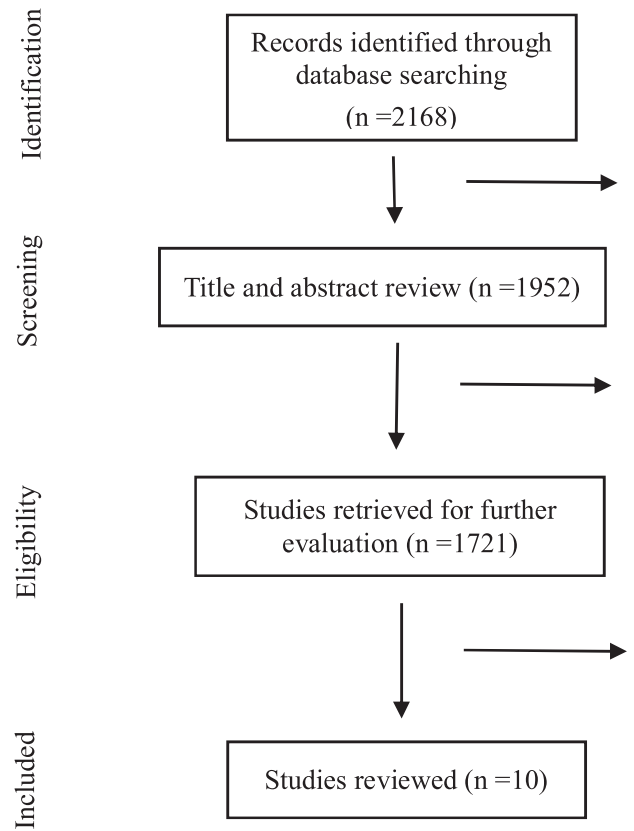

Excluded duplicates $(\mathrm{n}=216)$

Excluded : $(\mathrm{n}=231)$

Review articles: 194

Letter: 19

Comment: 18

Fig. 1. Flowchart of the study.

2019), and the maximal score was $42.3 \pm 3.5$ points (van Groenestijn, et al., 2019). The lowest FVC of lung function was $80.7 \% \pm 10.0 \%$ (Plowman, et al., 2019), and the highest FVC was $101.19 \% \pm 17.9 \%$ (Clawson, et al., 2018). Respiratory training intervention were active inspiratory exercise programs (Pinto, et al., 2012) and expiratory muscle strength training (Plowman, et al., 2019), and physical exercise interventions were resistance exercise (Dal BelloHaas, et al., 2007); cardiopulmonary exercise testing (Braga, et al., 2018); resistance, endurance, or stretching/range of motion exercise (Clawson, et al., 2018); moderate physical exercise program (Drory, et al., 2001); strictly monitored exercise programs (Lunetta, et al., 2016); aerobic exercise therapy (Merico, et al., 2018; van Groenestijn, et al., 2019); and intensive exercise regimen (Zucchi, et al., 2019). The shortest and longest intervention durations were $5.3 \pm 6.9$ and 30.2 \pm 11.8 months, respectively, after ALS diagnosis (van Groenestijn, et al., 2019; Merico, et al., 2018).

\subsection{Methodological quality of included trials}

The methodological quality of the included trials is shown in Table 2 and Fig. 2. The quality was assessed using the Cochrane risk of bias tool (RoB 2.0). The study by Braga et al. (2018) was considered to have two high-risk biases: bias due to deviations from intended interventions and bias due to missing outcome data (incomplete outcome data). Patients from Group $1(n=24)$ in their study performed aerobic exercise with controlled intensity; all patients underwent cardiopulmonary exercise tests (CPETs). Among patients from Group $2(n=24)$ who received standard care, six patients underwent CPET. Six months later (end of the study protocol), all patients from Group 1 completed the exercise program, but only $19(79 \%)$ had independent gait at the second CPET evaluation; six patients from Group 2 underwent the first CPET, and only five of those underwent the second CPET. The study by Drory et al. (2001) was considered to have a high risk of bias due to deviations from intended interventions, because all patients in both groups showed marked deterioration during the follow-up period and the drop-out rate was high.

\subsection{Outcomes}

Based on the availability of complete research data of the included trials, only five RCTs were included in the meta-analysis. Data were imputed as the SD of change from the baseline in each group and are presented as an average difference; a more negative difference indicated a worse result. 
Table 1

Characteristics of the included studies

\begin{tabular}{|c|c|c|c|c|c|c|}
\hline Author [year] & $\begin{array}{r}\text { Inclusion } \\
\text { criteria }\end{array}$ & $\begin{array}{l}\text { Patient } \\
\quad(\text { male, } \%)\end{array}$ & Age $(M \pm \mathrm{SD})$ & $\begin{array}{l}\text { Severity } \\
\text { ALSFRS-R } \\
(M \pm \text { SD }) \\
\end{array}$ & Intervention and Method & $\begin{array}{l}\text { Outcome } \\
\text { measure }\end{array}$ \\
\hline \multicolumn{7}{|c|}{ Respiratory training: } \\
\hline Pinto [2012] & $\begin{array}{l}\text { ALS } \\
\text { Age } 18-75 \text { ys } \\
\text { Disease }<24 \text { ms } \\
\text { ALSFRS }>24 \\
\text { FVC }>70 \%\end{array}$ & $\begin{array}{l}\text { E: 13(54) } \\
\text { C: } 13(85)\end{array}$ & $\begin{array}{l}\text { E: } 57.1 \pm 9.3 \\
\text { C: } 56.8 \pm 8.7\end{array}$ & $\begin{array}{l}\text { E: } 34.4 \pm 3.6^{*} \\
\text { C: } 33.5 \pm 3.8\end{array}$ & $\begin{array}{l}\text { E: active IMT protocol } 2 \\
\text { times/ day for } 8 \text { months } \\
\text { (threshold load was set to } \\
30-40 \% \text { of the MIP) } \\
\text { C: a placebo exercise } \\
\text { program for the first } 4 \\
\text { months and then active } \\
\text { exercise for the second } \\
\text { 4-month period }\end{array}$ & $\begin{array}{l}\text { ALSFRS } \\
\text { FSS } \\
\text { FIM } \\
\text { FVC } \\
\text { MEP } \\
\text { PEF } \\
\text { MVV }\end{array}$ \\
\hline $\begin{array}{c}\text { Plowman } \\
\text { [2019] }\end{array}$ & $\begin{array}{l}\text { ALS } \\
\text { FVC }>65 \% \\
\text { ALSFRS-R }>30\end{array}$ & $\begin{array}{l}\text { E: 24(71) C: } \\
24(50)\end{array}$ & $\begin{array}{l}\text { E: } 63.1 \pm 10.0 \\
\text { C: } 60.1 \pm 10.3\end{array}$ & $\begin{array}{l}E: 36.6 \pm 6.3 \\
C: 37.5 \pm 6.1\end{array}$ & $\begin{array}{l}\text { E: Expiratory muscle } \\
\text { strength training (EMST) } \\
\text { for } 8 \text { weeks (set at } 50 \% \text { of } \\
\text { pt's MEP) } \\
\text { C: sham EMST (the internal } \\
\text { spring removed) }\end{array}$ & $\begin{array}{l}\text { ALSFRS-R } \\
\text { FVC } \\
\text { MEP } \\
\text { PEF } \\
\text { Swallowing }\end{array}$ \\
\hline \multicolumn{7}{|c|}{ Physical exercise: } \\
\hline Braga [2018] & $\begin{array}{l}\text { Age } 18-90 \text { ys } \\
\text { Disease } 6-24 \mathrm{~ms} \\
\text { ALSFRS- } \\
\quad \mathrm{R} \geq 30 \\
\text { FVC } \geq 70 \%\end{array}$ & $\begin{array}{l}\text { E: 24(75) } \\
C: 24(59)\end{array}$ & $\begin{array}{l}\text { E: } 63.2 \pm 13.0 \\
\text { C: } 62.0 \pm 12.1\end{array}$ & $\begin{array}{l}\text { E: } 40.4 \pm 5.0 \\
C: 37.4 \pm 4.9\end{array}$ & $\begin{array}{l}\text { E: Aerobic Exercise } \\
\text { Controlled Intensity } \\
\text { (AECI) ( } 2 \text { times/ week on } \\
\text { a treadmill, training zone } \\
\text { determined by CPET; } \\
\text { moderate intensity for } 6 \\
\text { months)+SC } \\
\text { C: standard care }\end{array}$ & $\begin{array}{l}\text { ALSFRS-R } \\
\text { FVC } \\
\mathrm{VO}_{2} \\
\mathrm{VCO}_{2} \\
\mathrm{VE}\end{array}$ \\
\hline $\begin{array}{r}\text { Clawson } \\
\text { [2018] }\end{array}$ & ALS & $\begin{array}{l}\text { E1:21(71) } \\
\text { E2:18(50) } \\
\text { E3:20(75) }\end{array}$ & $\begin{array}{l}\text { E1:57.7 } \pm 9.7 \\
\text { E2:63.7 } \pm 10.6 \\
\text { E3:57.8 } \pm 11.9\end{array}$ & $\begin{array}{l}\text { E1:39.7 } \pm 3.7 \\
\text { E2:39.2 } \pm 4.9 \\
\text { E3:39.6 } \pm 5.0\end{array}$ & $\begin{array}{l}\text { E1: Stretching exercise } \\
\text { (SROM) for } 6 \text { months } \\
\text { E2: Resistance exercise } \\
\text { (weightlifting: weights for } \\
\text { upper and lower limbs, } \\
\text { determined by 1RM each } \\
\text { week) } \\
\text { E3: Endurance exercise } \\
\text { (stationary bicycling: } \\
\text { cycling for upper and } \\
\text { lower lims) }\end{array}$ & $\begin{array}{l}\text { Tolerability } \\
\text { ALSFRS-R } \\
\text { FVC } \\
\text { FSS } \\
\text { ALSQoL } \\
\mathrm{VO}_{2 \max }\end{array}$ \\
\hline $\begin{array}{l}\text { Dal } \\
\text { Bello-Haas } \\
\text { [2007] }\end{array}$ & $\begin{array}{l}\text { Early stage ALS } \\
\text { FVC }>90 \% \\
\text { ALSFRS }>30\end{array}$ & $\begin{array}{l}E: 13 \\
C: 14\end{array}$ & NP & NP & $\begin{array}{l}\text { E: resistance exercises (6 } \\
\text { months, } 2-3 \text { times/week } \\
\text { stretching, (in muscle } \\
\text { strength grade }>3 \text {, with } \\
\text { moderate load)+UC } \\
\text { C: usual care (once daily } \\
\text { stretching exercises) }\end{array}$ & $\begin{array}{l}\text { ALSFRS } \\
\text { FSS } \\
\text { SF-36 } \\
\text { FVC } \\
\text { MVIC }\end{array}$ \\
\hline Drory [2001] & $\begin{array}{l}\text { Mild-moderate } \\
\text { stages ALS } \\
\text { Age } 41-80 \text { ys }\end{array}$ & $\begin{array}{l}\text { E: 14(57) } \\
\text { C: 11(55) }\end{array}$ & $\begin{array}{l}\text { E: } 58.0 \pm 13.2 \\
\text { C: } 60.7 \pm 16.4\end{array}$ & $\begin{array}{l}\text { E and } \\
\text { C: Mean 27.5* }\end{array}$ & $\begin{array}{l}\text { E: moderate daily exercise } \\
\text { program ( } 15 \text { mins/time, } \\
2 \text { times/day) for } 6 \text { months } \\
\text { C: only usual daily life } \\
\text { requirement, no physical } \\
\text { activity }\end{array}$ & $\begin{array}{l}\text { ALSFRS } \\
\text { FSS } \\
\text { SF-36 } \\
\text { Visual analogue } \\
\quad \text { scale }\end{array}$ \\
\hline Lunetta [2016] & $\begin{array}{l}\text { Mild-moderate } \\
\text { disability ALS } \\
\text { Disease } \leq 24 \mathrm{~ms} \\
\text { Age } 18-75 \text { ys } \\
\text { FVC } \geq 70 \%\end{array}$ & $\begin{array}{l}\text { E: } 30(70) \\
(1 \mathrm{~A}-10,1 \mathrm{~B}-10, \\
1 \mathrm{C}-10) \\
\mathrm{C}: 30(57)\end{array}$ & $\begin{array}{l}\text { E: } 61.1 \pm 10.1 \\
\text { C: } 60.3 \pm 9.9\end{array}$ & $\begin{array}{l}\mathrm{E}: 39.1 \pm 4.7 \\
\mathrm{C}: 38.3 \pm 5.1\end{array}$ & $\begin{array}{l}\text { E: strictly monitored exercise } \\
\text { programs (SMEP) (daily } \\
\text { for } 2 \text { weeks/month, for } 6 \\
\text { months, three subgroups) } \\
\text { C: usual care (passive } \\
\text { exercise in } 6 \text { muscle for } \\
\text { 20mins and stretching } \\
\text { 2days/week) }\end{array}$ & $\begin{array}{l}\text { ALSFRS-R } \\
\text { FVC } \\
\text { MGQoL } \\
\text { Survuval }\end{array}$ \\
\hline
\end{tabular}


Table 1

(Continued)

\begin{tabular}{|c|c|c|c|c|c|c|}
\hline Author [year] & $\begin{array}{c}\text { Inclusion } \\
\text { criteria }\end{array}$ & $\begin{array}{l}\text { Patient } \\
\quad \text { (male, \%) }\end{array}$ & Age $(M \pm \mathrm{SD})$ & $\begin{array}{l}\text { Severity } \\
\text { ALSFRS-R } \\
(M \pm \text { SD })\end{array}$ & Intervention and Method & $\begin{array}{l}\text { Outcome } \\
\text { measure }\end{array}$ \\
\hline \multicolumn{7}{|c|}{ Physical combined aerobic and resistance training: } \\
\hline Merico [2018] & $\begin{array}{l}\text { Mild-moderate } \\
\text { disability ALS }\end{array}$ & $\begin{array}{l}\text { E: 23(57) } \\
\text { C: } 15(67)\end{array}$ & $\begin{array}{l}\text { E: } 61.6 \pm 10.6 \\
\text { C: } 59.8 \pm 14.7\end{array}$ & $\begin{array}{l}\text { E: } 36.1 \pm 4.7 \\
\text { C: } 34.5 \pm 3.6\end{array}$ & $\begin{array}{l}\text { E: ALS-EP (progressive } \\
\text { training muscle strength } \\
\text { and aerobi endurance, } \\
\text { daily for } 5 \text { weeks, aerobic } \\
\text { intensity of } 65 \% \text { of MHR, } \\
\text { duration of } 15-20 \text { minutes) } \\
\text { C: ALS-SNT (standard } \\
\text { neuromotor rehabilitation } \\
\text { treatment) (consisting } 1 \\
\text { hour of stretching, active } \\
\text { mobilization and general } \\
\text { muscle reinforcement) }\end{array}$ & $\begin{array}{l}\text { FSS } \\
\text { FIM } \\
6 \mathrm{MWT} \\
\mathrm{MRCSS} \\
\mathrm{VO}_{2} \\
\end{array}$ \\
\hline $\begin{array}{l}\text { Van } \\
\text { Groenestijn } \\
\text { [2019] }\end{array}$ & $\begin{array}{c}\text { Ambulatory } \\
\text { ALS Age } \\
18-80 y s \\
\text { FVC }>80 \%\end{array}$ & $\begin{array}{l}\text { E: } 27(67) \\
\text { C: } 30(73)\end{array}$ & $\begin{array}{l}\text { E: } 60.9 \pm 10.0 \\
\text { C: } 59.9 \pm 10.7\end{array}$ & $\begin{array}{l}E: 42.3 \pm 3.5 \\
C: 42.3 \pm 4.2\end{array}$ & $\begin{array}{l}\text { E: Aerobic exercise therapy } \\
\text { (AET)+usual care } \\
\text { (consisted of a 16-week } \\
\text { aerobic cycling exercise } \\
\text { program) } \\
\text { C: usual care }\end{array}$ & $\begin{array}{l}\text { ALSFRS-R } \\
\text { CIS-Fatigue } \\
\text { FVC } \\
\text { SF-36 } \\
\text { ALSAQ-40 }\end{array}$ \\
\hline Zucchi [2019] & $\begin{array}{l}\text { ALS Disease } \\
\leq 18 \mathrm{~ms} \\
\text { Age } 18-86 \mathrm{ys} \\
\text { FVC }>50 \%\end{array}$ & $\begin{array}{l}\text { E: 32(81) } \\
\text { C: } 33(70)\end{array}$ & $\begin{array}{l}\text { E: } 65.14 \pm 9.9 \\
\text { C: } 64.7 \pm 10.1\end{array}$ & $\begin{array}{l}E: 39.8 \pm 5.7 \\
C: 40.2 \pm 5.2\end{array}$ & $\begin{array}{l}\text { E: intensive exercise regimen } \\
\text { (IER, } 5 \text { sessions/week, } 50 \\
\text { session) } \\
\text { C: usual exercise regimen } \\
\text { (UER, } 2 \text { sessions/week, } 20 \\
\text { sessions) (Exercise } \\
45 \text { mins/session for } 10 \\
\text { week. } 1 \text {.aerobic training on } \\
\text { treadmill or cyclette, with } \\
\text { moderate (Borg3) intensity, } \\
\text { total } 30 \text { mins. } 2 . \text { endurance } \\
\text { training, on non-affected } \\
\text { muscles, } 40 \% \text { of MCV, } \\
\text { 12-15 reps per } 2 \text { sets. } 3 . \\
\text { Stretching or assisted } \\
\text { active mobilization) }\end{array}$ & $\begin{array}{l}\text { ALSFRS-R } \\
\text { FVC } \\
\text { FSS } \\
\text { ALSAQ-40 } \\
\text { MGQoL } \\
\end{array}$ \\
\hline
\end{tabular}

ALS: amyotrophic lateral sclerosis; *ALSFRS: ALS functional rating scale (0-40); ALSFRS-R: ALS functional rating scale-revised (0-48); ALSAQ-40:40-item ALS assessment questionnaire; CBT: cognitive behavioral therapy; CPET: cardiopulmonary exercise testing; FIM: functional independence measure; FSS: fatigue severity scale(9-63); FVC: forced vital capacity; IMT: inspiratory muscle training; MEP: maximum expiratory pressure; MGQoL: McGill Quality of Life; MIP: maximal inspiratory pressure; MRCSS: MRC sum score; MVIC: maximum voluntary isometric contraction; MVV: maximal voluntary ventilation; PEF: peak expiratory flow; SF-36: Short Form-36; VO 2 : oxygen uptake; $\mathrm{VCO}_{2}$ : carbon dioxide output; VE: minute ventilation; 6MWT: six minute walk test; NP: not provided.

Table 2

Methodological quality assessment of included trials

\begin{tabular}{lcccccc}
\hline Study [year] & Allocation bias & Performance bias & Attrition bias & Detection bias & Reporting bias & Overall risk of bias \\
\hline Pinto [2012] & Low risk & Some concerns & Low risk & Low risk & Low risk & Low risk \\
Plowman [2019] & Low risk & Some concerns & Low risk & Low risk & Low risk & Low risk \\
Braga [2018] & Some concerns & High risk & High risk & Low risk & Low risk & Some concerns \\
Clawson [2018] & Low risk & Low risk & Low risk & Some concerns & Low risk & Low risk \\
Dal Bello-Haas [2007] & Low risk & Some concerns & Low risk & Low risk & Some concerns & Low risk \\
Drory [2001] & Low risk & High risk & Some concerns & Some concerns & Some concerns & Some concerns \\
Lunetta [2016] & Low risk & Some concerns & Low risk & Some concerns & Some concerns & Low risk \\
Merico [2018] & Low risk & Some concerns & Low risk & Some concerns & Some concerns & Low risk \\
Van Groenestijn [2019] & Low risk & Some concerns & Low risk & Low risk & Low risk & Low risk \\
Zucchi [2019] & Low risk & Some concerns & Low risk & Some concerns & Some concerns & Low risk \\
\hline
\end{tabular}




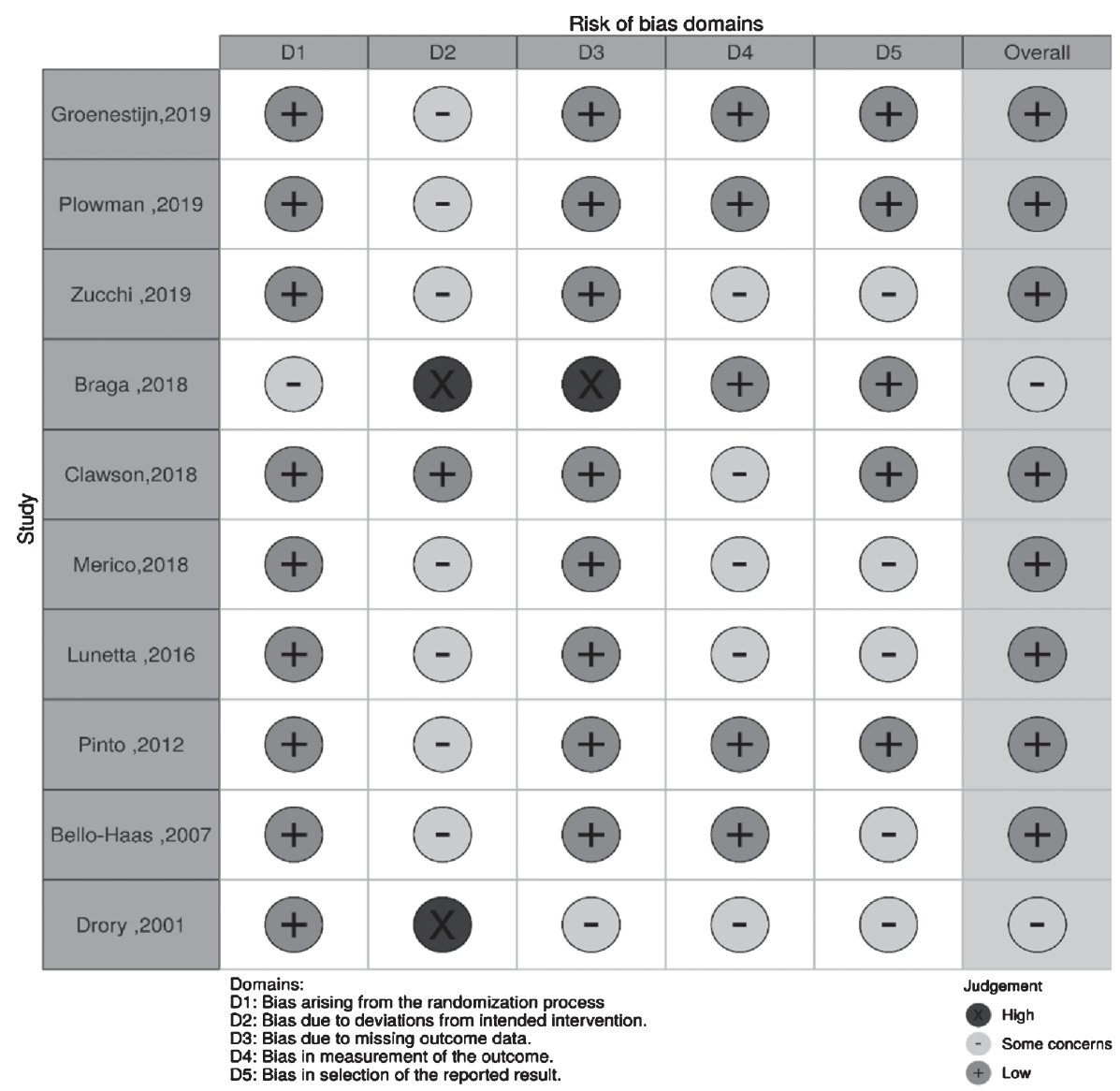

Bias arising from the randomization process Bias due to deviations from intended interventions Bias due to missing outcome data Bias in measurement of the outcome Bias in selection of the reported result Overall risk of bias

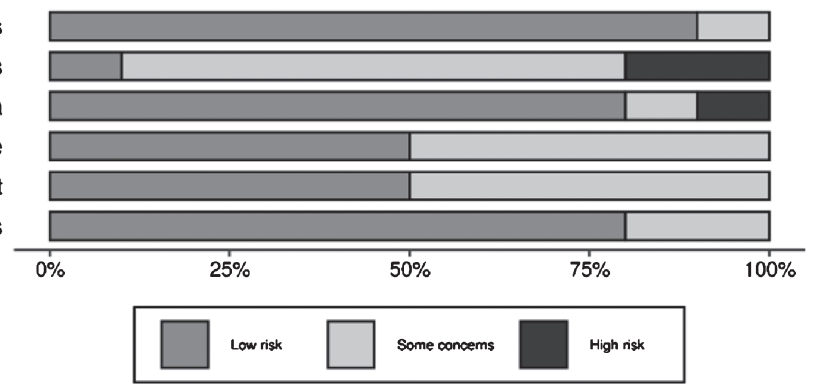

Fig. 2. Judgments about each risk of bias item for each included trial.

\subsection{ALSFRS-R}

In the two RCTs involving respiratory trainings, the one by Pinto et al. (2012) evaluated functional status using the ALSFRS score (0-40), whereas that by Polwman et al. (2019) used the ALSFRS-R score (0-48). Pinto et al. assessed the ALSFRS score at 4 and 8 months after intervention, whereas Polwman et al. assessed the ALSFRS-R score at 8 weeks after intervention. No difference was observed the
ALSFRS-R scores at 2-4 months after intervention between patients who received respiratory trainings and those who received usual care $(1.63 ; 95 \% \mathrm{CI}$ : -0.78 to $4.04 ; I^{2}$ : $0 \%$; Fig. 3). Moreover, Pinto et al. observed that the ALSFRS scores did not differ significantly (MD: $0.70 ; 95 \%$ CI: -3.08 to 4.48 ) between the respiratory exercise and usual care groups at 8 months after intervention.

Three studies involving physical exercise were included in the meta-analysis (Braga, et al., 2018; 


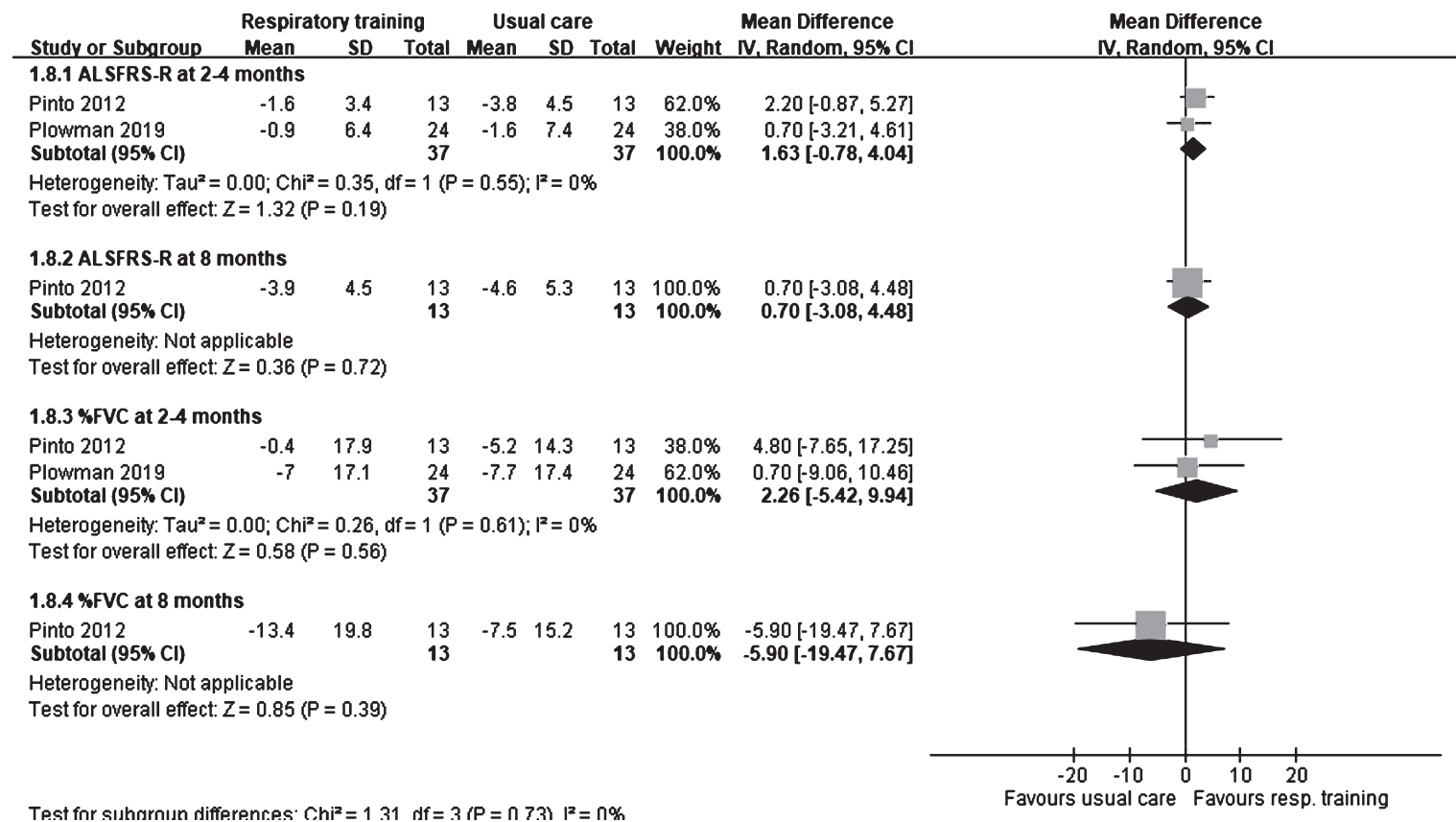

Fig. 3. Forest plot of respiratory training vs. usual care. Outcome: ALSFRS-R and \%FVC.

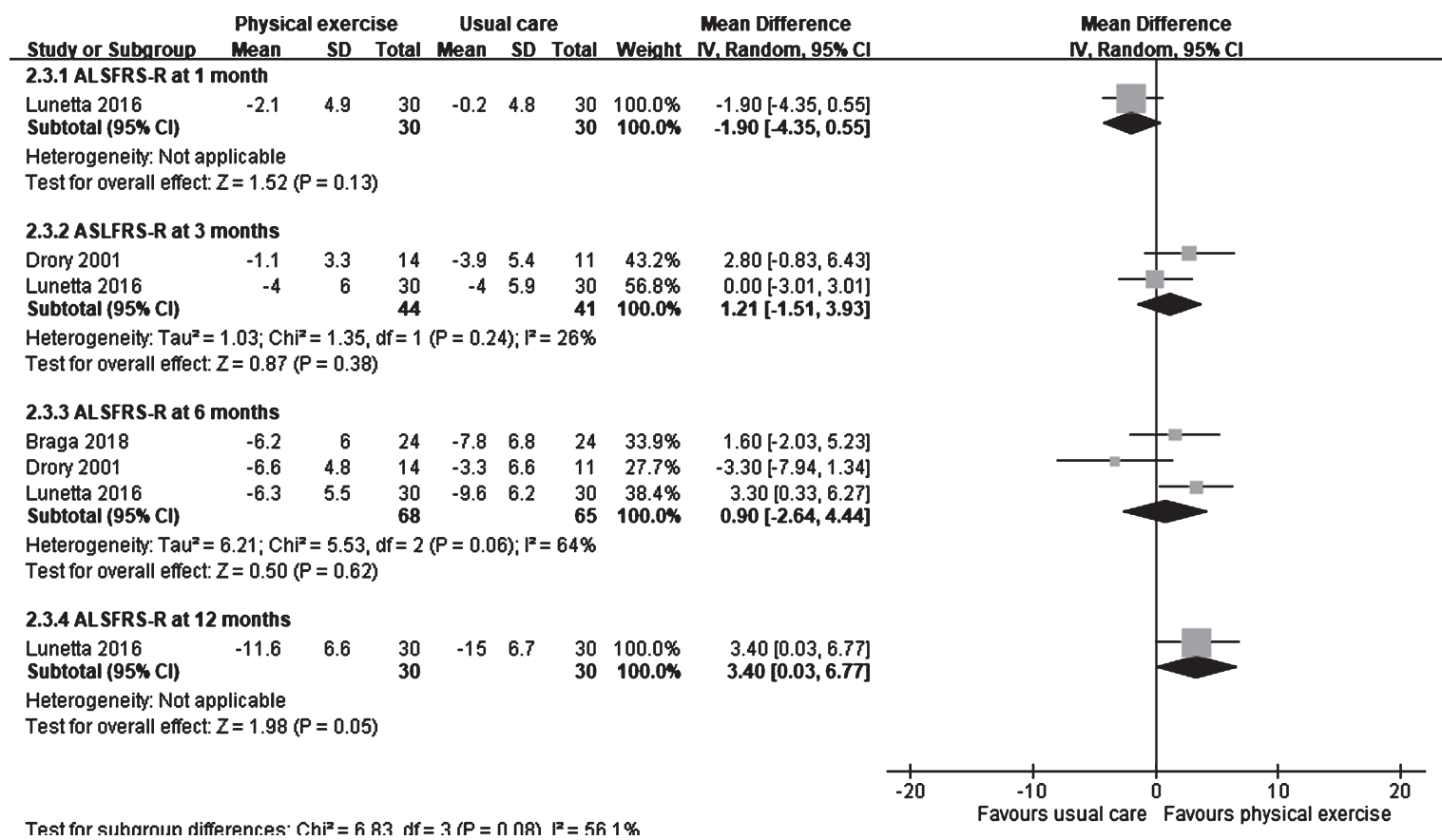

Fig. 4. Forest plot of physical exercise vs. usual care. Outcome: ALSFRS-R.

Drory, et al., 2001; Lunetta, et al., 2016). Lunetta et al. observed that the ALSFRS-R score in the usual care group was higher than that in the physical exercise group (MD: $-1.90 ; 95 \% \mathrm{CI}:-4.35$ to 0.55 ) at 1 month after intervention (Fig. 4). No difference was observed in the score between the groups at 3 months $\left(1.21 ; 95 \%\right.$ CI: -1.51 to $3.93 ; I^{2}: 26 \%$ ) (Drory, et al., 2001; Lunetta, et al., 2016) and 6 months (0.90; 
95\% CI: -2.64 to $4.44 ; I^{2}: 64 \%$ ) after intervention (Braga, et al., 2018; Drory, et al., 2001; Lunetta, et al., 2016). However, at 12 months after intervention, the ALSFRS-R score in the physical exercise group was higher than that in the usual care group (MD: 3.40; 95\% CI: 0.03 to 6.77) (Lunetta, et al., 2016).

\section{6. $\% F V C$}

$\% \mathrm{FVC}$ was assessed in two trials that involved respiratory training (Pinto, et al., 2012; Plowman, et al., 2019). At 2-4 months after intervention, no difference was found in $\% \mathrm{FVC}$ between the respiratory training and usual care groups $(2.26 ; 95 \% \mathrm{CI}:-5.42$ to $9.94 ; I^{2}: 0 \%$; Fig. 3 ). Even at 8 months after intervention, Pinto et al. observed that \%FVC did not differ significantly (MD: $-5.90 ; 95 \% \mathrm{CI}:-19.47$ to 7.67) between the respiratory training and usual care groups.

\section{Discussion}

Our meta-analysis demonstrated no difference in the ALSFRS-R score and \%FVC among patients with ALS regardless of whether they received the respiratory training or physical exercise intervention. In addition, there was little difference in intervention effectiveness over time. At 12 months after intervention, Lunetta et al. (2016) observed that the ALSFRS-R score in the physical exercise group was higher than that in the usual care group. They used strictly monitored exercise programs (SMEPs), and exercise was limited by patients' fatigue (7/10 on the Borg perceived scale and $75 \%$ of the predicted heart rate). The patients were trained daily for 2 weeks every month for 6 months. They were divided into three subgroups: SMEP 1, in which the patients performed upper- and lower-limb exercises on a cycloergometer each for 20 minutes and active exercise involving six muscle groups with strength $>3$ MRC (three reps/set for each muscle); SMEP 2, where patients performed active exercise involving six muscle groups with strength $>3 \mathrm{MRC}$ (three reps/set for each muscle); and SMEP 3, where patients performed passive exercises involving six muscle for 20 minutes. Although the exercise program caused no significant change in $\% \mathrm{FVC}$ and had no effect on patient survival, Lunetta et al. found that SMEPs may significantly reduce motor deterioration in patients with ALS. They speculated that the frequency and intensity of the exercise plan might affect disease progression.

Although currently no cure or drug exists for treating ALS, many studies focused on methods to delay the progression of the disease. Several studies reported that exercise is helpful for rehabilitation. Increasing number of animal and human studies showed that exercise programs, especially moderateintensity endurance exercise, can delay the onset of the disease and increase survival rates (Mahoney, et al., 2004; Majmudar, et al., 2014; Pinto, et al., 2012; WHO, 2020). Therefore, exercise, including stretching, balance, range of motion, and physical activity, has become a part of the standard care of patients with ALS. However, the role of exercise in patients with ALS has been controversial. Highly repetitive exercises or heavy resistance exercises may cause long-term loss of weakness or loss of neuromuscular strength.

The included trials were divided into three categories based on the exercise intervention involved: respiratory training, physical exercise, and physical exercise involving a combination of aerobic and resistance training.

Respiratory training: In the studies by Pinto et al. (2012) and Plowman et al. (2019), no change was observed in the ALSFRS-R score or \%FVC regardless of whether the patients received inspiratory or expiratory muscle training; this trend persisted even when the intervention was performed over a long period (Pinto, et al., 2012). However, some improvements were observed in the minimal and maximal inspiratory pressure and peak expiratory flow.

Physical exercise: Only three of the five studies that involved physical exercise were included in our meta-analysis. No positive effect on the ALSFRS$\mathrm{R}$ score or \% FVC was observed in patients with ALS in these studies. Thus, although physical exercise could still improve the ALSFRS-R score, the difference may not be clinically significant between the groups. In all these studies, patients underwent physical exercise training for 6 months.

Combination of aerobic and resistance training: Three studies involved a combination of aerobic exercises and resistance training; although the meta-analysis lacked sufficient data regarding these studies, we could analyze them individually. These studies (Merico, et al., 2018; van Groenestijn, et al., 2019; Zucchi, et al., 2019) also showed that exercise training did not significantly affect ALSFRS-R scores and respiratory function. Only Merico et al. showed that exercise protocol could improve physical 
activity and muscle power. Their exercise protocol was performed for 15-20 minutes daily for 5 weeks, whereas the exercise program was performed for 16 and 10 weeks in the studies by van Groenestijn et al. and Zucchi et al., respectively. However, the exercise training methods classified in these studies did not improve the lung function of patients.

In terms of safety, Clawson et al. (2018) reported that resistance, endurance, and stretching exercises are safe and do not deteriorate the function of patients with ALS. Moreover, patients who performed resistance training and stretching exercises showed better continuity in the 6-month training. Van Groenestijn et al. (2019) also showed that pure aerobic exercises have no side effects in patients with ALS, although little changes in ALSAQ-40 (40-item ALS assessment questionnaire), SF-36, mental component summary, and physical component summary were observed. That is, the quality of life of patients was still the same after 6 months of intervention. Further, patients who did not withdraw from the study and completed the training were tracked for 10 months; their ALSFRS$\mathrm{R}$ scores had improved, and they lived longer than the patients who withdrew from the study did. Existing evidence shows that muscle strengthening and cardiovascular exercises can at least help maintain function and do not adversely affect disease progression in patients with ALS.

The included RCTs had high heterogeneity that was due to the different methods of rehabilitation intervention. However, we divided the studies into three categories. Hence, our meta-analysis showed high homogeneity. Studies that involved respiratory trainings had low heterogeneity $\left(I^{2}=0 \%\right)$. Studies that involved physical exercises showed low heterogeneity $\left(I^{2}=26 \%\right)$ in the ALSFRS-R score at 3 months after intervention. However, the 6-month ALSFRS-R score showed substantial heterogeneity $\left(I^{2}=64 \%\right)$ among the studies. We attributed this difference to the different training frequencies in the different studies despite the total training time being 6 months. The training frequency in the studies by Drory et al. (2001) and Lunetta et al. (2016) was twice a day and once a day for 2 weeks every month, respectively. The training frequency in another study was twice a week (Braga, et al., 2018).

Our study has some limitations. First, the rehabilitation intervention methods differed among the studies, and we classified them first to reduce heterogeneity. Second, some studies did not have complete data; thus, they could not be included in the meta-analysis. Third, owing to the limited available information, our meta-analysis focused on only two outcomes, namely ALSFRS-R and \%FVC.

\section{Conclusion}

In this study, we aimed to determine whether respiratory training or physical exercise affects the ALSFRS-R score and lung function of patients with ALS. Our meta-analysis demonstrated that exercise intervention exerted no appreciable effects on the ALSFRS-R score and lung function. Further clinical trials are warranted to develop approaches for improving the lung function of patients with ALS. However, we still found no strong evidence showing the potential harmful effect of rehabilitation exercise in ALS.

\section{Conflict of interest}

None to report.

\section{References}

Braga, A. C. M., Pinto, A., Pinto, S., \& Carvalho, M. (2018). The Role of Moderate Aerobic Exercise as Determined by Cardiopulmonary Exercise Testing in ALS. Neurology Research International, Article ID 8218697, pp. 10. https://doi.org/ 10.1155/2018/8218697

Brown, R. H., \& Al-Chalabi, A. (2017). Amyotrophic lateral sclerosis. New England Journal of Medicine, 377, 162-172.

Cedarbaum, J. M., Stambler, N., Malta, E., Fuller, C., Hilt, D., Thurmond, B., \& Nakanishi, A. (1999). The ALSFRS-R: a revised ALS functional rating scale that incorporates assessments of respiratory function. BDNF ALS Study Group (Phase III). Journal of the Neurological Sciences, 169(1-2), 13-21. doi: 10.1016/s0022-510x(99)00210-5

Cheah, B. C., Boland, R. A., Brodaty, N. E., Zoing, M. C., Jeffery, S. E., McKenzie, D. K., \& Kiernan, M. C. (2009). INSPIRATIonAL-INSPIRAtory muscle training in amyotrophic lateral sclerosis. Amyotroph Lateral Sclerosis, 10(5-6), 384-392.

Clawson, L. L., Cudkowicz, M., Krivickas, L., Brooks, B. R., Sanjak, M., Allred, P., Atassi, N., Swartz, A., Steinhorn, G., Uchil, A., Riley, K. M., Yu, H., Schoenfeld, D. A., \& Maragakis, N. J. (2018). A randomized controlled trial of resistance and endurance exercise in amyotrophic lateral sclerosis. Amyotrophic Lateral Sclerosis and Frontotemporal Degeneration, 19(3-4), 250-258.

Dal Bello-Haas, V., Florence, J. M., Kloos, A. D., Scheirbecker, J., Lopate, G., Hayes, S. M., Pioro, E. P., \& Mitsumoto, H. (2007). A randomized controlled trial of resistance exercise in individuals with ALS. Neurology, 68, 2003-2007. 
DerSimonian, R., \& Laird, N. (1986). Meta-analysis in clinical trials. Control Clinical Trials, 7, 177-88.

Drory, V. E., Goltsman, E., Reznik, J. G., Mosek, A., \& Korczyn, A.D. (2001). The value of muscle exercise in patients with amyotrophic lateral sclerosis. Journal of the Neurological Sciences, 191, 133-137.

Ferreira, G. D., Costa, A. C. C., Plentz, R. D. M., Coronel, C. C., \& Sbruzzi, G. (2016). Respiratory training improved ventilatory function and respiratory muscle strength in patients with multiple sclerosis and lateral amyotrophic sclerosis: systematic review and meta-analysis. Physiotherapy, 102, 221-228.

Gosselink, R., Kovacs, L., Ketelaer, P., Carton, H., \& Decramer, M. (2000). Respiratory muscle weakness and respiratory muscle training in severely disabled multiple sclerosis patients. Archives of Physical Medicine and Rehabilitation, 81(6), 747-751.

Kiernan, M. C., Vucic, S., Cheah, B. C., Turner, M. R., Eisen, A., Hardiman, O., Burrell, J. R., \& Zoing, M. C. (2011). Amyotrophic lateral sclerosis. Lancet, 377(9769), 942-955. doi:10. 1016/s0140-6736(10)61156-7

Liberati, A., Altman, D. G., Tetzlaff, J., Mulrow, C., Gotzsche, P. C., Ioannidis, J. P., Clarke, M., Devereaux, P. J., Kleijnen, J., \& Moher, D. (2009). The PRISMA statement for reporting systematic reviews and meta-analyses of studies that evaluate healthcare interventions: explanation and elaboration. $B M J$, 339, b2700. https://doi.org/10.1136/bmj.b2700

Lunetta, C., Lizio, A., Sansone, V. A., Cellotto, N. M., Maestri, E., Bettinelli, M., Gatti, V., Melazzini, M. G., Meola, G., \& Corbo M. (2016). Strictly monitored exercise programs reduce motor deterioration in ALS: preliminary results of a randomized controlled trial. Journal of Neurology, 263, 52-60. DOI 10.1007/s00415-015-7924-z

Mahoney, D. J., Rodriguez, C., Devries, M., Yasuda N., \& Tarnopolsky, M. A. (2004). Effects of high-intensity endurance exercise training in the G93A mouse model of amyotrophic lateral Sclerosis. Muscle \& Nerve, 29(5), 656-662.

Majmudar, S., Wu, J., \& Paganoni, S. (2014). Rehabilitation in amyotrophic lateral sclerosis: Why it matters. Muscle \& Nerve, 50(1), 4-13.

Merico, A., Cavinato, M., Gregorio, C., Lacatena, A., Gioia, E., Piccione, F., \& Angelini, C. (2018). Effects of combined endurance and resistance training in Amyotrophic Lateral Sclerosis: A pilot, randomized, controlled study. European Journal of Translational Myology, 28(1), 132-140.

Pinto, S., \& de Carvalho, M. (2013). Can inspiratory muscle training increase survival in early-affected amyotrophic lateral sclerosis patients? Amyotrophic Lateral Sclerosis and Frontotemporal Degeneration, 14(2), 124-6.
Pinto, S., Swash, M., \& Carvalho, M. D. (2012). Respiratory exercise in amyotrophic lateral sclerosis. Amyotrophic Lateral Sclerosis, 13, 33-43. DOI: 10.3109/17482968.2011.626052

Plowman, E. K., Tabor-Gray, L., Rosado, M., Vasilopoulos, T., Robison, R., Chapin, J. L., Gaziano, J., Vu, T., \& Gooch, C. (2019). Impact of expiratory strength training in amyotrophic lateral sclerosis: Results of a randomized, sham-controlled trial. Muscle Nerve, 59, 40-46. DOI 10.1002/mus.26292

Rochester, C. L., Vogiatzis, L., Holland, A. E., Lareau, S. C., Marciniuk, D. D., Puhan, M. A., Spruit, M. A., Masefield, S., Casaburi, R., Clini, E. M., Crouch, R., Garcia-Aymerich, J., Garvey, C., Goldstein, R. S., Hill, K., Morgan, M., Nici, L., Pitta, F., Ries, A. L., ... ZuWallack, R. L. (2015). An Official American Thoracic Society/European Respiratory Society Policy Statement: Enhancing Implementation, Use, and Delivery of Pulmonary Rehabilitation. American Journal of Respiratory and Critical Care Medicine, 192(11), 1373-1386.

Sterne, J. A. C., Savović, J., Page, M. J., Elbers, R. G., Blencowe, N. S., Boutron, I., Cates, C. J., Cheng, H.-Y., Corbett, M. S., Eldridge, S. M., Emberson, J. R., Hernán, M. A., Hopewell, S., Hróbjartsson, A., Junqueira, D. R., Jüni, P., Kirkham, J. J., Lasserson, T., Li, T., ... Higgins, J. P. T. (2019). RoB 2: a revised tool for assessing risk of bias in randomised trials. BMJ, 366, 14898. https://doi.org/10.1136/bmj.14898

van Es, M. A., Hardiman, O., Chio, A., Al-Chalabi, A., Pasterkamp, R. J., Veldink, J. H., \& van den Berg, L. H. (2017). Amyotrophic lateral sclerosis. Lancet, 390(10107), 2084-2098.

van Groenestijn, A. C., Schröder, C. D., van Eijk, R. P. A., Veldink, J. H., Kruitwagen-van Reenen, E. T., Groothuis, J. T., Grupstra, H. F., Tepper, M., van Vliet, R. O., Visser-Meily, J. M. A., \& van den Berg, L. H. (2019). Aerobic Exercise Therapy in Ambulatory Patients With ALS: A Randomized Controlled Trial. Neurorehabilitation and Neural Repair, 33(2), 153-164. DOI: $10.1177 / 1545968319826051$

World Health Organization (WHO). (2020, Nov 26). Physical activity Fact Sheet. https://www.who.int/news-room/factsheets/detail/physical-activity

Zucchi, E., Vinceti, M., Malagoli, C., Fini, N., Gessani, A., Fasano, A., Rizzi, R., Sette, E., Cavazza, S., Fiocchi, A., Buja, S., Faccioli, T., Storani, S., \& Mandrioli, J. (2019). High-frequency motor rehabilitation in amyotrophic lateral sclerosis: a randomized clinical trial. Annals of Clinical and Translational Neurology, 6(5), 893-901. DOI: 10.1002/acn3.765 